\title{
Doctor-Patient Relationship and Multimorbidity: The More Extraordinary a Case Seems, the Easier it is to Solve it
}

\author{
Jose Luis Turabian* \\ Specialist in Family and Community Medicine, Health Center Santa Maria de Benquerencia, Regional Health Service of \\ Castilla la Mancha (SESCAM), Toledo, Spain
}

\begin{abstract}
An effective patient-doctor communication and trust become especially important in managing patients with two or more chronic conditions. Multimorbidity, which, moreover, is more frequent in the elderly patient, is one of the many factors that affect doctor-patient communication and trust. Older patients care with multimorbidity implies the creation of a different type of doctor-patient relationship. Multimorbidity and especially in the elderly patient, is an extraordinarily complex matter; but the patient-doctor relationship facilitates understanding, simplification and consensual intervention. So, in this article we hypothesize that it is the relationship and communication with the patient the key to solving the complexity of these patients. The main elements that should have this type of relationship and communication with the elderly patients with multimorbidity are: 1) Focus on the specific patient and its context; 2) Focus on prioritizing problems with "energy" (the "master problems"); 3) Focus on the patient's life history and continuity; 4) Focus on integrality; 5) Focus on the prognosis (which is marked by psycho-social factors); and, 6) Focus on simplification (reduce the team of professionals who care for the patient and pharmacological des-prescription).. The doctor-patient relationship in multimorbidity is a simple and powerful tool to address it. In the absence of evidence-based clinical guidelines, better patient-doctor communication is required to overcome the challenges of managing multiple chronic conditions. It has to think with Allan Poe that "the more extraordinary a case seems, the easier it is to solve it".
\end{abstract}

\section{Keywords}

Multimorbidity, Physician-patient communication, General practice, Framework, Physician-patient relations, Communication training, Chronic disease

\footnotetext{
"The more extraordinary a case seems, the easier it is to solve it"
}

\section{Edgar Allan Poe}

\section{Introduction}

Chronic diseases contribute a large share of disease burden in low-middle-income and in high-income countries. Chronic diseases have a tendency to occur simultaneously and where there are two or more such conditions, this is termed as "multimorbidity" [1]. It is a highly prevalent health problem, and it is associated to negative health consequences, such as a poorer quality of life and functional status, higher rates of hospital admission and avoidable readmissions, decreased quality of life, functional decline, and increased healthcare utilisation, including emergency admissions, particularly with higher numbers of coexisting conditions, may reduce adherence, produce conflicts in treatment, and is not yet supported by evidence-based clinical recommendations [2-5].

On the other hand, multimorbidity occurs preferably in the elderly patient. Thus, these patients have special characteristics regarding communication and doctor-patient rela- tionship [6]. Due to the aging of the population and due to the improvements in medical care, there is a life extension and so, it is more common for the older people to have to deal with more diseases (i.e. osteoarthritis, arterial hypertension, cardiovascular disease, diabetes, depression, COPD, loss of visual and auditory capacity, falls and immobility, osteoporosis, dementia, delirium, urinary incontinence, etc.). So, based on a series of studies (although with non-homogeneous diagnostic criteria), the prevalence rates in the elderly range from $55 \%$ to $98 \%[5,7]$.

Further, common chronic conditions in older people clus-

\footnotetext{
*Corresponding author: Jose Luis Turabian, Specialist in Family and Community Medicine, Health Center Santa Maria de Benquerencia, Regional Health Service of Castilla la Mancha (SESCAM), Toledo, Spain, ORCID iD: 0000-0002-8463-171X

Accepted: May 20, 2019

Published online: May 22, 2019

Citation: Turabian JL (2019) Doctor-Patient Relationship and Multimorbidity: The More Extraordinary a Case Seems, the Easier it is to Solve it. Arch Fam Med Gen Pract 4(1):82-89
} 
ter together in broad categories. These broad clusters are qualitatively distinct and are important predictors of several health and functioning outcomes [8]. Complex patients (i.e. older people with multimorbidity) are increasingly common in general medicine and often have poor clinical outcomes. Healthcare system barriers to effective care for complex patients have been previously described, as specific psychosocial factors, financial constraints, logistical challenges, physical limitations, lifestyle changes, emotional impact, inadequate family and social support, and the complexity of managing multiple conditions, medications, and communicating with health care providers, but less is known about the potential impact and meaning of caring for complex patients on a daily basis for general practitioner (GP) [9].

GPs are increasingly obliged to care for elderly people with complex health problems. But, care for patients with multimorbidity is a challenging task for the GP because: 1) Multimorbidity may produce conflicts in treatment decisions because of indications and contra-indications for a specific treatment are often found in the same person; 2) Multimorbidity is not yet supported by evidence-based clinical recommendations; and 3) Because of this complexity, multimorbidity may reduce patients' adherence to treatment plans [5].

On the other hands, doctor-patient relationship has been and remains a keystone of care [10]. Patient-doctor relationship has been defined as a meaningful therapeutic interaction between the patients and their doctors [11].

The doctor-patient relationship is a complex phenomenon conformed by several aspects, among which we can highlight the doctor-patient communication, the patient's participation in the decision making and the patient's satisfaction. These characteristics have been associated with the communication behavior of the doctor and the autonomy of the patient in medical care. The transcendence of the doctor-patient relationship is given by the confirmed fact of its influence on the results of health care. In fact, the quality of doctor-patient interaction and communication is a powerful indicator of the quality of medical care and plays a fundamental role in the medical care process [12-14].

Multi-morbidity receives considerable attention in public policy, health and social care, but not regarding its influence on the doctor-patient relationship. However, the intersection of elderly patient care with multimorbidity and the patientphysician relationship is the key point to solve these extraordinarily complex cases: for example, the presence in the same older patient of osteoarthritis, arterial hypertension, cardiovascular disease, diabetes, depression, COPD, loss of visual and auditory capacity, falls and immobility, osteoporosis, dementia, delirium, urinary incontinence, etc., and also, financial constraints, emotional impact, inadequate family and social support, and polypharmacy, and communication difficulties with health care providers, etc. In this context, this article tries to conceptualize the main elements of the doctorolder patient relationship with multimorbidity at the level of general medicine.

\section{Discussion}

\section{Multimorbidity is an increasing challenge}

Findings highlight the challenge of multimorbidity both in high income and in low- and middle-income countries, and particularly among the lower socioeconomic groups, and so, there is a pressing need for reorientation of health care resources considering the distribution of multimorbidity and its adverse effect on health outcomes [1].

In resource-limited settings as well in high income countries, having multiple conditions, especially in the elderly, are associated with limitations in daily living, depression and poor health, especially among those with lower socioeconomic status, highlighting the need for interventions to reduce multimorbidity [15].

The multi-morbidity is "infinite", and in this way it is presented for the GP as non-operative to make useful decisions. Thus, patients with multi-morbidity produce feelings of despair and impotence in the GP: To their problems it seems that the known patterns of the diseases and existing protocols and guides can not be applied because of their comorbidity. Intensity and complexity of these problems can overwhelm even experienced professionals. So, in many cases, the attention is made entirely in somatic terms or using biological protocols, which if applied to each of the patient's problems, produce contradictions and originate new and serious problems such as polypharmacy [16].

Further, "hassles" are frequently reported by patients with multimorbidity in primary care, including multiple appointments, poor co-ordination, and conflicting recommendations, lack of information about conditions and treatment options, poor communication among health professionals, and poor access to specialist care [17].

\section{Doctor-patient relationship}

Meaningful therapeutic interaction between patients and doctors includes both patient-doctor communication and trust. An effective patient-doctor communication consists of explaining patient's illnesses and treatments, spending adequate time, managing uncertainty, and developing rapport [11].

Person-centered relationship: In the professional interaction patient-physician, the existence of an effective communication and the satisfaction of the patient with this relationship take on considerable importance. It has been observed that providing the necessary information in a way that favours and guarantees minimum levels of understanding on the part of the patient contributes to improving adherence. Similarly, the satisfaction of the patient, from the affective point of view, with the established relationship with the therapist has been associated with a marked increase in therapeutic compliance. Thus, this aspect highlights the importance of the medical-patient therapeutic alliance, which in the initial instance will constitute the first element of fortification of the patient's compliance with the medical recommendation and by both will have a considerable influence on the therapeutic process and in the final results of treatment. Without an ad- 
equate alliance from the beginning of therapy, adherence is less likely to subsist [18-21].

Doctor-patient relationship is fundamental for the diagnosis and for the treatment: The doctor-patient relationship is fundamental both for the diagnosis and for the treatment. All health care activity is influenced, directly or indirectly, by the interpersonal relationship. Communication allows the integration of clinical reasoning by connecting the biomedical and psychosocial aspects of clinical care. The interview is a technique or a channel and place of communication, where the patient-doctor relationship is produced and developed. The doctor's relationship with the patient is the true core of clinical practice in general medicine [22].

Doctor-patient communication allows the doctor to elaborate the bio-psycho-social meaning of the symptom: $\mathrm{GP}^{\prime}$ active listening in clinical interview allows the doctor to elaborate the bio-psycho-social meaning of the symptom, that is, the elaboration of a kind of cartography or map of the patient, and of the doctor: the symptom as seen by the doctor and the prognosis of evolution of this symptom, and on the other hand, the perception of the patient with what he feels and thinks has changed in his life by this symptom, as well as his forecast of what will happen next, his defence mechanisms, the structure of his personality, his socio-labour and familiar situation, his idea of pharmacological treatment, his motivation, his expectation of the result, of the adverse effects, the participation of caregivers or relatives, and the anticipation of change of the sentinel symptom with respect to the efficacy of the drug $[23,24]$.

Types of doctor-patient relationships: The transcendence of the doctor-patient relationship is given by the confirmed fact of its influence on the results of health care. Several models of doctor-patient relationship can be described, as: 1. "Paternalistic"; 2. "Consumerist"; and 3. "Mutual", but evidence of improved compliance, satisfaction and recall of physician information has been found in patient-centered consultations. Since these concepts of doctor-patient relationship and patient-centered consultation have multiple facets, they are complex to understand and teach. Using a metaphor is a tool that can be useful in these situations. We could say that the "good" doctor-patient relationship is a process where an "alliance" is created: A process in which the doctor adapts to the rhythm of the patient and little by little can help him move towards healthier scenarios; that is, detect what dance the patient dances and like a good dancer, take a step back, another forward, dancing and pacing with the patient. But there is not a single type of "good" or "adequate" doctor-patient relationship; there is not "a single dance that the patient dances" [12,25].

Doctor-patient relationship is especially important in the management of patients with multimorbidity: The doctor-patient relationship implies different decision-making models (diagnostic and treatment). Since the symptoms are subjective evidences of health problems, and are expressed differently according to the context of the doctor-patient relationship, this variable limits the degree to which the physician obtains psychosocial information from the patient, and involves different diagnoses, and treatment $[26,27]$.

In addition, for the proper understanding of the reason for consultation, the GP usually needs to pass from the material manifested by the patient initially, to the latent and unexpressed material. In this way, the GP can transform a slightly understandable material into a broader, more comprehensive and more comprehensible material. In this basic process, which begins the preparation of the diagnosis, the relationship that the patient tends to create with the doctor has an important meaning $[28,29]$.

So, an effective patient-doctor communication and trust become especially important in managing patients with two or more chronic conditions (i.e., multimorbidity). Multimorbidity affects elderly population disproportionately and is projected to increase from 34,835 million in 2000 to 81,999 million by 2050 [11].

An effective communication and high trust with the doctor can improve the care of the elderly with multimorbidity. A special case of multimorbidity arises when an individual has coexisting chronic physical and mental health conditions. Presence of mental health condition in addition to chronic physical conditions can be very challenging for disease management. Effective communication and trust have been shown to improve outcomes for not only individuals with chronic physical conditions but also those with a mental health condition [11].

However, according to the previous studies, factors other than multimorbidity can affect meaningful therapeutic interactions between the patients and their doctors (doctorpatient relationship). These may include age, gender, race/ ethnicity, socioeconomic status, health status, and length of the visit [11].

But, it can be said that the main factors or variables that influence the doctor-patient relationship are: 1) Those due to the doctor; 2) Those due to the patient (personal characteristics, including age); 3) Those due to the disease (such as multimorbidity); 4) Those due to the drug that is prescribed or not in the consultation (such as polypharmacy, and the need for de-prescription in these patients); 5) Those due to the continuity of the relationship; and 6) Those due to the type of health system or service (elderly patients with multimorbidity are usually treated by numerous healthcare providers, such as internist, geriatrician, and various specialists such as endocrinologist, cardiologist, etc., social worker, nurse, etc., in hospital admissions and in outpatient consultations).

A more active and patient-centered role has been proposed in which there is greater control of the patient, a reduction in the doctor's domain and greater mutual participation, and has now become the predominant model in the clinical practice. But, interventions to improve patientcentered care for persons with multimorbidity are in constant growth [30].

In this context, patient care with multimorbidity, therefore, implies the creation of a different type of doctor-patient relationship. Furthermore, it is the relationship and communication with the patient that is the key to solving the com- 
Citation: Turabian JL (2019) Doctor-Patient Relationship and Multimorbidity: The More Extraordinary a Case Seems, the Easier it is to Solve it. Arch Fam Med Gen Pract 4(1):82-89

Table 1: Main elements that must be taken into account in the relationship and communication with the patient with multimorbidity.

\begin{tabular}{|l|l|}
\hline 1 & -Focus on the specific patient and its context \\
\hline 2 & -Focus on prioritizing problems with "energy" or "hot" with qualitative methodology (and these "hot" problems are not the most \\
serious pathologies, or the most important, etc.): These are the "master problems".
\end{tabular}

plexity of these patients. The main elements that should have this type of relationship and communication with the elderly patient with multimorbidity are (Table 1) [31]:

A) Focus on the specific patient and its context

B) Focus on prioritizing problems with "energy" or "hot" with qualitative methodology (and these problems are not the most serious pathologies, or the most important, etc.): These are the "master problems".

C) Focus on the continuity and history of the patient's life

D) Focus on integrality

E) Focus on the prognosis, which is marked by psychosocial factors

F) Focus on simplification: Reduce the team of professionals who care for the patient and in pharmacological prescription

\section{A) Focus on the specific patient and its context}

Guideline developers should be aware of the complexity of multimorbidity. As a consequence of this complexity, it is even more important to focus on what matters to a patient with multimorbidity in general practice [7].

The elderly patient with multimorbidity expects the following from the doctor-patient relationship: 1) Someone providing him with a trusting relationship; 2) Someone assisting him; 3) Someone who is on his side. So, it is important to establishing trusting relationships between older persons and their GPs in order to provide truly assistance. The older persons valued the GP acting as informed but unbiased facilitators [32].

There is a broad international consensus that multimorbidity is best addressed in primary care settings by a patient-centred approach, including regular appointments for comprehensive problem review and management options tailored to individual patient preferences. This care should be provided by a multidisciplinary team with a named lead family doctor/general practitioner [31,33].

Doctor's appearing to be in a hurry during the visit is perceived as an uncaring attitude by the patients. Patients place importance on having a GP who talks at a slow pace and allows the patient to take his time. It is especially critical for an elderly patient with multiple chronic conditions because elderly patients and their families have to take care of all the co-occurring physical and mental health conditions at the same time and may want to talk about all of their diagnoses and comprehend numerous prescribed drugs and treatments. It is important for the clinicians not to hurry during consultation with an elderly patient having multimorbidity and allow the patient to take his own time to understand their multiple diagnoses and treatments [11].

Arriving at a diagnosis sometimes seems to prevail over other needs and may bias the care and the doctorpatient relationship. When the GP, in the care of the elderly patient focuses on reaching certain diagnostic categories of standardized classification, it is probably forgetting everything that that person saves as a particular person.

The GP must take into account the need for a symmetrical colloquium in the clinical interview with the elderly patient with multimorbidity, para que the doctor pueda to listen and integrate to what the elderly person wants to communicate, including being attentive to non-verbal communication. GP also have to calibrate his own language and make feedback of what the patient expressed. The interview with the elderly with multimorbidity is a means for him to reconstruct his life, where the meaning of care will emerge and perhaps also the factors and conditions that can be identified in the roots of a pathology in the context of a clinical picture.

Thus, doctor-patient relationship in elderly with multimorbidity, does not refer mainly to a contact between sick and professional to restore the health of the former, but a contact with a specific or particular patient, with a life experience [6].

B) Focus on prioritizing "hot" problems or problems with "energy", preferably using qualitative methodology: These are the "master problems" (and remember that these "master problems" could be not the most serious pathologies or the biological most important problems)

Multimorbidity a challenge to GPs. It is difficult for addressing the competing demands of multiple physical and mental conditions in busy outpatient settings. Discussion of multiple illnesses and treatments within a short visit may leave some patients feeling that the doctor did not discuss or provide all the information regarding their conditions and treatments [11].

This doctor-patient relationship centered on the specific patient and their life history leads to a common understanding of the "master problems". A "master" problem is one in which, in the judgment of the GP and the patient, for that patient at that time and in that context, it concentrates the greatest importance or significance for the patient's health/ 
illness, and allows us to "move forward" ("Open doors"), making it easier to unblock a situation, change or move from one scenario to another with new perspectives or restore interrupted connections ("Re-connect").

Finding the "main problem" is a subjective experience. How to determine that one problem has more value or energy than another? How to determine what is a "master problem"? Using a hidden rule, by which we will make an analysis, and which is like the entire scientific operation, subject to verification and improvement: The "master problem" is one that, 1) Has a character of importance, and is more permanent, deep and elementary; 2) A convergence of its effects is observed that intensifies its value up to the highest limit; and 3) The intervention on that problem is more beneficial or adequate $[16,31]$. life

C) Focus on the continuity and history of the patient's

Complex health systems make it difficult for older persons with multimorbidity to achieve continuity of care [32]. The available data suggest that elderly people benefit from a long-term continuing doctor-patient relationship, and are markedly loyal to their doctor. It has been reported that 55\% of elderly people stayed with the same doctor 5 years or more, and $36 \%$ ten years or more. The benefits of this long continuity of care and the doctor-patient relationship that it entails, achieves less healthcare costs, improves patient satisfaction, and reduces poor quality healthcare approaches [34].

With better understanding of lived experiences of patients, GP may advance patient care and medical education. In the continuity of care five themes appear: 1) Goals of care and decision making; 2 ) Complexity; 3 ) Meeting expectations; 4) Logistics; and 5) Interpersonal dynamics. The complexities of multimorbidity lead to shared feelings of vulnerability, uncertainty and enforced compromises. Barriers to optimal care and education included system constraints, inadequate continuity and role uncertainty [35].

The long doctor-patient relationship allows a more comprehensive management of its multiple problems, the substitution of costly hospital care for other of lower cost at the ambulatory level, early knowledge of changes in their biopsychosocial situation, and a more integral preventive and educational intervention [34].

Multimorbidity in old people is usually chronic, and unlike what can happen in previous stages of life, in the elderly tends to interfere more significantly in their personal and relational life. This chronic multimorbidity in the elderly tends to destroy their self-sufficiency, and in this situation the disease in the elderly is an expression of a complex and polymorphous underground discomfort. There may be loneliness, isolation and economic precariousness, which favor the appearance of discomforts of different nature, with possible somatic manifestations. The multimorbidity in the elderly patient tends to create important psychological connotations different from those of other stages of life. One of the elder's biggest concerns is the loss of self-sufficiency.
The disease can cause fear of chronicity and disability, which intensifies the emotional problem, and the connotation of abandonment, marginalization and poverty.

This situation has repercussions in the doctor-patient relationship, where the GP that can not have as a goal the "restitutio ad integrum" (the complete restoration). So, GP must focus not on the complete remission of the patient symptoms, but on achieving a good level of autonomy with sufficient quality. For the elderly patient with multimorbidity, the idea of health is generally associated with the recovery of a certain degree of self-sufficiency rather than the resolution of the chronic disease that he has accepted over time and with which he has learned to live.

The flow of this problem can cause distress and depression, and varied reactions on the last stage of his life, including somatic responses and physical pain, all of which aggravates his disability and the manifest pathology. The elderly with multimorbidity presents a fertile ground for psychosomatic disorders, and the evolution of their organic diseases has a multifactorial genesis, including psychological ones.

These peculiarities suggest to the GP a relationship with the sick old man that is oriented in a longitudinal and current vision of the physical symptoms that are presented, but also and especially in the history and personal experience of each person $[6,36,37]$.

\section{D) Focus on integrality}

The word Integral means to integrate, to bring together; to have a comprehensive vision of the patient is to take into account the interrelationships of the different dimensions of a person and see the whole as greater than the sum of its parts, as well as the acceptance of integration, or the whole, as a fact. Comprehensive care in general medicine must address the biological, psychological and social aspects of each patient. Integrality in general medicine also means that family doctor is committed to the total person rather than to a particular body of knowledge, group of diseases or a special technique. In the practice of family medicine, integrality implies the understanding that the observer is an integral part of what he is observing. Thus, the focus is on collaboration (including our patients), the co-evolution of knowledge and health patterns [38].

Most people with long term health problems have more than one condition, and, for many, multiple conditions are the norm. In stark contrast, the healthcare system is organised to deal with single conditions and to separate mental and physical healthcare, complicating attempts to provide integrated care for both aspects of health [2-5].

The high prevalence of multimorbidity and numerous combinations of conditions suggests that single, diseaseoriented management programs may be less effective or efficient tools for high quality care compared to personcentered approaches [39]. A majority of GPs perceive the chief complaints of patients of the multimorbid patients correctly, but there is room for improvement [40].

When there is a disease that seems to be from the 
dominant or priority biomedical point of view, there is a risk that this pathology will attract all the attention of the GP, and other problems that can be very important for the elderly patient will not be taken into consideration [41]. Elderly patient with multimorbidity, more than any other, requires a unified evaluation of his disease, and when possible, the support and integration of his own family [6].

E) Focus on the prognosis, which is marked by psychosocial factors

The conventional clinical approach implies to establish a diagnosis, a prognosis and a treatment. "Prognosis" is the forecast of the probable outcome or course of a disease; the patient's chance of recover. Diagnosis has been considered as the main guideline for treatment and is considered the central component of clinical practice; but prognosis, which refers to the possible outcomes of a disease and the frequency with which they can be expected to occur, is an essential element of health care decision making is related to "what is going to happen next".

The prognosis is a fundamental requirement to be able to make correct therapeutic decisions. Many are the factors depending on the disease, such as its severity and extent, the involvement of vitally important organs, the degree of disorder in the organic functions and the possibility of reversibility of the functional and morphological changes of the organism, the frequency and severity of its complications and the duration of the disease, which greatly influence the prognosis, and should be known and evaluated. Other factors that related to the patient, such as age, sex, previous health status, the diseases they suffer, the inheritance, the cultural level, the disposition and possibilities of cooperating with the treatment, and the physical and mental state are also very important in the forecast. The temperament and character of the patient are also significant for the prognosis of the course of the disease and the ability to work after convalescence. Some patients become disabled because of insignificant disorders, and others fight valiantly against their illness and have an active lifestyle.

GPs are able to understand the prognosis and full impact of multimorbidity (neurological, cardiologic, etc.) and assess the prognosis, course and severity in a specific individual, without having "protocols" for each health problem in each patient. What do family doctors use to target the breadth of the clinic? How do they manage to find the middle of the clinic, without apparent reference points, in such concrete data? The course of the disease is influenced by the concurrent psychosocial factors; the severity and prognosis of the disease can be predicted more accurately from the loss of social and family roles and activities than from the biologic pathology. The prognosis of diseases depends more on psychosocial factors that of biomedical factors. Patient prognosis is determined by more than disease diagnosis. Prognosis can be highly variable in persons with a same particular diagnosis. There are different prognoses of the same symptom or disease according to contexts [31,42].

F) Focus on simplification: Reduce the team of professionals who care for the patient and in pharmacological pre-

\section{scription}

Multidisciplinarity and teamwork are fundamental in the care of the elderly patient with multimorbidity. Multimorbidity also affects care coordination and leads to poor management of co-occurring conditions due to the focus on one dominant chronic condition [11]. The GP must lead the patient's care team, and reduce this team to the minimum possible expression to improve the results.

On the other hand, the management of multimorbidity with drugs is often complex, resulting in polypharmacy with its attendant risks. Effective management of care and medication regimens for complex patients remains an unresolved challenge. Patients with multimorbidity have a high treatment burden in terms of understanding and self managing the conditions, attending multiple appointments, and managing complex drug regimens. Qualitative research highlights the "endless struggle" patients experience in trying to manage their conditions well [2-5].

The tendency to increase in prescriptions and polypharmacy leads to a consequence of iatrogenesis. Although this problem has different levels of reflection, these comments focus on the micro level of the GP consultation. There is a technical and ethical need to rationalize pharmacological prescription, and that includes pharmacological non-prescription of drugs when they are not indicated (for example, antibiotics for viral infections) and pharmacological de-prescription when indicated (for example, opioid coincidence and benzodiazepines, or statins as primary prevention in the elderly, etc.), which must be made by the GP., although there may be many difficulties, both with respect to the doctor, and to the possible pressures of the patient to have the drug. Here, pharmacological non-prescription and pharmacological de-prescription are one of the main factors that alter the doctor-patient relationship [43].

It must be remembered that giving a medication is the tangible expression of attending and giving help. Thus, the prescription is a complex issue; it is a ritual. There are many elements in this exchange: Promise and expectation of giving and receiving, of instructing, of evolving, of caring for and accepting care, and others, that are not within the active ingredients of pharmacology. The prescription indicates relationship. For every specific patient, the style of this transaction must be accommodated to ensure the success of the relationship $[6,7]$. So, the medicine most frequently used by GPs is the doctor himself [32].

The pharmacological prescription and the interview for pharmacological non-prescription and pharmacological de-prescription can give rise to a certain model of doctor-patient relationship that is more focused on the drug than on other more significant aspects of the medical intervention. Somehow, polypharmacy, but also the attempt to avoid pharmacological prescriptions leads to "another communication or relationship only with the drug", where the doctor is absent (and his effects: The "doctor in itself as a drug") [44].

\section{Conclusion}

With multimorbidity being a norm in the elderly popu- 
lation, policy focus has recently shifted towards the better illness management of these individuals. Elderly patients with multimorbidity need care that is responsive to the interactions between multiple conditions and treatments and is tailored to their individual needs. An effective communication and high trust between the patient and doctor are necessary for improving the care of these patients.

Communication and trust are container concepts and comprise several different domains. And, doctor-older patient relationship and communication has special nuances and characteristics [45]. Patients' perceived communication and trust with the doctor might be influenced by several other factors such as the severity of patient's chronic condition, differences in patient and providers' culture and communication styles, and organizational factors. Multimorbidity is, therefore, one of the many factors that affect patient-doctor communication and trust.

In short, multimorbidity and especially in the elderly patient, it's a complex issue, we could say "extraordinarily" complex issue; but we know that the presence of multimorbidity affects the doctor-patient relationship, and that this patient-doctor relationship facilitates understanding, simplification and consensual intervention. So, the doctor-patient relationship with multimorbidity, at the same time, it is a simple and powerful tool to address it. You have to think with Edgar Allan Poe that "the more extraordinary case seems, the easier it is to solve it" [46]. With or without evidence-based clinical guidelines (which, moreover, do not exist now), better patient-doctor communication is required to overcome the challenges of managing multiple chronic conditions. As if it were an extraordinary story by Edgar Allan Poe, the clues to solve the multimorbidity are in doctor-patient relationship.

\section{References}

1. Arokiasamy $P$, Uttamacharya U, Jain K, et al. (2015) The impact of multimorbidity on adult physical and mental health in lowand middle-income countries: What does the study on global ageing and adult health (SAGE) reveal? BMC Med 13: 178.

2. Wallace E, Salisbury C, Guthrie B, et al. (2015) Managing patients with multimorbidity in primary care. BMJ 350: 176.

3. Gunn J (2015) Designing care for people with mixed mental and physical multimorbidity. BMJ 350: 712.

4. Hansen RA, Voils $\mathrm{Cl}$, Farley JF, et al. (2015) Prescriber continuity and medication adherence for complex patients. Ann Pharmacother 49: 293-302.

5. Scherer M, Hansen H, Gensichen J, et al. (2016) Association between multimorbidity patterns and chronic pain in elderly primary care patients: A cross-sectional observational study. BMC Fam Pract 17: 68.

6. Cesa-Bianchi M, Critini C, Cesa-Bianchi G (2000) Anziani e comunicazione tra salute e malatia. Guidotti, Milano.

7. Sinnige J, Korevaar JC, Westert GP, et al. (2015) Multimorbidity patterns in a primary care population aged 55 years and over. Fam. Pract 32: 505-513.

8. Olaya B, Moneta MV, Caballero FF, et al. (2017) Latent class analysis of multimorbidity patterns and associated outcomes in Spanish older adults: A prospective cohort study. BMC Geriatr
17: 186.

9. Loeb DF, Bayliss EA, Candrian C, et al. (2016) Primary care providers' experiences caring for complex patients in primary care: A qualitative study. BMC Fam Pract 17: 34.

10. Turabian JL (2019) Doctor-patient relationships: A puzzle of fragmented knowledge. J Family Med Prim Care Open Access 3: 128.

11. Garg R, Shen C, Sambamoorthi N, et al. (2016) Type of multimorbidity and patient-doctor communication and trust among elderly medicare beneficiaries. Int J Family Med 2016: 8747891.

12. Turabian JL (2018) Doctor-patient relationship as dancing a dance. Journal of Family Medicine 1: 1-6.

13. Beck RS, Daughtridge R, Sloane PD (2002) Physician-patient communication in the primary care office: A systematic review. J Am Board Fam Pract 15: 25-38.

14. Matusitz J, Spear J (2014) Effective doctor-patient communication: An updated examination. Soc Work Public Health 29: 252-266.

15. Jackson CA, Jones M, Tooth L, et al. (2015) Multimorbidity patterns are differentially associated with functional ability and decline in a longitudinal cohort of older women. Age Ageing 44: 810-816.

16. Turabián JL, Pérez Franco B (2016) A way of helping "Mr. Minotaur" and "Ms. Ariadne" to exit from the multiple morbidity labyrinth: The "master problems". Semergen 42: 38-48.

17. Adeniji C, Kenning C, Coventry PA, et al. (2015) What are the core predictors of 'hassles' among patients with multimorbidity in primary care? A cross sectional study. BMC Health Serv Res 15: 255.

18. Niolu C, Siracusano A (2005) Discontinuità psicofarmacologica e aderenza. II Pensiero Scientifico Editore, Roma.

19. Dilla T, Valladares A, Lizán L, et al. (2009) Treatment adherence and persistence: Causes, consequences and improvement strategies. Aten Primaria 41: 342-348.

20. Martín Alfonso L (2004) Acerca del concepto de adherencia terapéutica. Rev Cubana Salud Pública 30: 350-352.

21. American Medical Association (2000) Doctor-patient relationship: The doctor-patient relationship in pharmacotherapy: Improving treatment effectiveness. JAMA 284: 2793-2794.

22. Turabian JL (2018) Doctor-patient relationship in pharmacological treatment: Discontinuation and adherence. COJ Rev \& Res 1: COJRR.000521.

23. Turabian JL, Perez Franco B (2011) Non-pharmacological aspects of medications. Semergen 37: 225-272.

24. Turabián JL, Pérez Franco B (2010) The concept of treatment in familiy medicine: A contextualised and contextual map of a city hardly seen. Aten Primaria 42: 253-254.

25. Turabián JL, Pérez Franco B (2008) The effect of seeing the sea for the first time. An Attempt at defining the family medicine law: The interview is clinical medicine. Aten Primaria 40: 565-566.

26. Turabian JL (2018) Doctor-patient relationship epidemiology and its implications on public health. Epidemol Int J 2: 000116.

27. Turabian JL (2018) The enormous potential of the doctor-patient relationship. Trends Gen Pract 1. 
28. Freud S (1966) Interpretation of dreams. Sigmund Freud Copyright, Ltd, London.

29. Balint M, Hunt J, Joyce D (1970) Treatment or diagnosis. A study of repeat prescriptions in general practice. Tavistock Publications, London.

30. Poitras ME, Maltais ME, Bestard-Denommé L, et al. (2018) What are the effective elements in patient-centered and multimorbidity care? A scoping review. BMC Health Serv Res 18: 446.

31. Turabian JL (2018) Notes for a theory of multimorbidity in general medicine: The problem of multimorbidity care is not in practice, but in the lack of theoretical conceptualization. Journal of Public Health and General Medicine 1: 1-7.

32. Hjelm M, Holst G, Willman A, et al. (2015) The work of case managers as experienced by older persons $(75+)$ with multimorbidity - a focused ethnography. BMC Geriatr 15: 168.

33. Dowrick C (2018) Patient-centred care for multimorbidity: An end in itself? Lancet 392: 4-5.

34. Netting FE, Williams FG (1999) Enhancing primary care of elderly people. Garland Publishing, Inc, New York.

35. Cottrell E, Yardley S (2015) Lived experiences of multimorbidity: An interpretative meta-synthesis of patients', general practitioners' and trainees' perceptions. Chronic IIIn 11: 279-303.

36. Turabian JL (2017) A narrative review of natural history of diseases and continuity of care in family medicine. Arch Community Med Public Health 3: 41-47.

37. Turabian JL (2017) Opportunistic prevention in family medicine: Anticipatory care, case-finding and continuity of care. J Health Care Prev 1: 101.
38. Turabian JL (2018) Concept of integrality in general medicine. Arch Fam Med Gen Pract 3: 54-57.

39. Pefoyo AJ, Bronskill SE, Gruneir A, et al. (2015) The increasing burden and complexity of multimorbidity. BMC Public Health 15: 415.

40. Neuner-Jehle S, Zechmann S, Grundmann Maissen D, et al. (2017) Patient-provider concordance in the perception of illness and disease: A cross-sectional study among multimorbid patients and their general practitioners in Switzerland. Patient Prefer Adherence 11: 1451-1458.

41. Subramaniam H (2019) Co-morbidities in dementia: Time to focus more on assessing and managing co-morbidities. Age Ageing 48: 314-315.

42. Turabian JL (2018) Prognosis-based medicine-the importance of psychosocial factors: Conceptualization from a case of acute pericarditis. Trends Gen Pract 1: 1-2.

43. Turabian JL (2018) Pharmacological non-prescription, doctorpatient relationship and biopsychosocial approach: Foreign lands or foreign travelers? Archives of Community and Family Medicine 1: 39-42.

44. Balint E, Norell JS (1973) Six minutes for the patient: Interactions in general practice consultations. Tavistock Publications, London.

45. Turabian JL (2019) Differential characteristics in communication and relationships of the general practitioner with the elderly patient. Journal of Family Medicine Forecast.

46. Aragón MA. Técnicas narrativas y suspense en el relato breve policíaco. AO XXXIX-XL.

DOI: $10.36959 / 577 / 487$

Copyright: (C) 2019 Turabian JL. This is an open-access article distributed under the terms of the Creative Commons Attribution License, which permits unrestricted use, distribution, and reproduction in any medium, provided the original author and source are credited. 